\title{
Optimum Threshold for SNR-based Selective Digital Relaying Schemes in Cooperative Wireless Networks
}

\author{
Furuzan Atay Onat ${ }^{1}$, Abdulkareem Adinoyi ${ }^{1}$, Yijia Fan $^{2}$, Halim Yanikomeroglu ${ }^{1}$, and John S. Thompson ${ }^{2}$ \\ ${ }^{1}$ Broadband Communications and Wireless Systems (BCWS) Centre, \\ Department of Systems and Computer Engineering Carleton University, Ottawa, Canada \\ e-mail: \{furuzan, adinoyi, halim\}@sce.carleton.ca \\ ${ }^{2}$ Institute for Digital Communications, School of Engineering and Electronics \\ University of Edinburgh, Edinburgh, UK \\ e-mail: \{y.fan, john.thompson\}@ed.ac.uk
}

\begin{abstract}
We study selective digital relaying schemes where the relay may choose to retransmit or to remain silent based on the qualities of the links between the source, relay and the destination. We first analyze a baseline scheme, called static relaying, where the relaying decisions are based only on the average signal-to-noise ratio (SNR) values of all the links. The second scheme, dynamic relaying, allows the relay to make decisions based on the instantaneous SNR of the source-relay link and average SNRs of the relay-destination and source-destination links. We show that, in dynamic relaying the optimal strategy to minimize the average end-to-end bit error rate is a threshold rule on the instantaneous SNR of the source-relay channel. In this case, the optimal threshold value is a function of average SNR of relay-destination and source-destination channels. We derive closed-form expressions for the optimal threshold and the bit error performance achieved by this threshold. We show that dynamic relaying can provide significant performance advantage over static relaying.
\end{abstract}

\section{INTRODUCTION}

It is well known that cooperation can increase reliability in wireless networks. Several protocols where users act as relays for each other are proposed in [1] and [2]. Diversity obtained this way is usually referred to as cooperative diversity. Depending on the signal processing performed by the relay, relaying schemes can be classified as analog or digital. In analog relaying the relay terminal amplifies the received signal and then retransmits. One disadvantage of analog relaying is the increased noise and interference. In digital relaying, the relay detects and possibly decodes the source signal and then regenerates and retransmits it, requiring more processing compared to analog relaying. The focus of this work is on digital cooperative relaying.

Cooperative relaying makes use of independent fading at source-destination and relay-destination channels. The destination is required to combine signals from different channels. In digital relaying, if the relay detection is correct, the destination

This work was supported by Wireless Tech. Labs, Nortel Networks. receives the signal through two branches and obtains diversity. However, if the relay has a detection error, the effective SNR at the destination after combining is reduced significantly. This phenomenon is called error propagation.

The error propagation limits the performance of the basic digital relaying protocol. One way of reducing error propagation is by using embedded error detection codes such that once an error is detected at the relay, the relay remains silent. While this idea is very effective in minimizing error propagation, it requires the relay to perform channel estimation, demodulation and then error detection for each data block before making a forwarding decision. These operations cause additional delay of the data even if the relay eventually decides not to transmit. Moreover, the relay node must be in either transmit or receive mode continuously during the data transfer. In wireless terminals, the amount of power consumed for receiving is negligible in comparison to the transmit power. However, the power required for receiving is significant in very low power devices such as sensor nodes.

A simpler way of reducing error propagation is to make forwarding decisions based on the received SNR at the relay. If the received SNR is low, the data is likely to have errors. Then, a relay node can go to "sleep mode" for durations in the scale of the coherence time of the channels between the relay and its neighbors.

The apparent trade-off between creating the required diversity branches to the destination and minimizing the risk of error propagation has motivated research on thresholdbased relaying ( [3]-[5]). The choice of the threshold has considerable impact on the end-to-end (e2e) performance of the cooperative diversity schemes. For instance, consider a relay detection threshold value of zero. This is akin to basic fixed digital relaying. It is known that the error propagation limits the diversity gain and the diversity order of such a single cooperation relay network is limited to one [1]. On the other hand, for a very high value of threshold setting, the system degenerates to one path channel, which is the source to destination channel and the dual diversity is not realized. 
In [3], the authors use a SNR threshold to determine if the source-relay channel is reliable and propose a heuristic formula to calculate the value of this threshold. The work in [4] studies the performance of threshold relaying in a multiantenna multi-relay architecture and shows the importance of the threshold especially when the relay has a small number of antennas. Both [3] and [4] tied the threshold selection to the average SNR of the source-relay channel. Although in symmetric networks, where the average SNRs of all the links are equal, the threshold should inevitably be a function of the average source-relay SNR, our results indicate that for arbitrary network configurations the optimal threshold is in fact independent of the the average source-relay SNR.

Another work on threshold relaying is presented in [5]. In this work the authors formulate the performance of thresholdbased relaying for an arbitrary threshold value. Then, they study the optimal threshold and power allocation numerically. However, they assume that the channel coefficients are real Gaussian random variables, which does not apply to typical wireless scenarios.

In [6], Lin et al. study the relay selection problem in the context of coded cooperation. They derive rules to decide whether using a particular relay is advantageous over retransmission by the source. The relay is selected to serve for a long period of time, which is larger than the scale of multi-path fading.

In this paper, we formulate the optimal threshold selection problem as a simple decision problem from the relay's point of view. We assume that the transmit power levels of the source and the relay are predetermined. We show that if the relay can dynamically choose to retransmit or to remain silent based on the instantaneous SNR of the source-relay channel, the optimal strategy to minimize the average bit error rate is a threshold rule and the optimal threshold value is a function of the average SNRs of relay-destination and source-destination channels. In order to illustrate the benefits of dynamic relaying we define another protocol, called static relaying, where the relaying decisions are based solely on the average SNR values of all the links.

\section{SyStem MOdEL}

In this paper, we consider a source $S$, a destination $D$ and a relay $R$, assisting $S-D$ communication. We assume a block fading channel model, where each channel stays the same during each block and the channel states at different blocks are i.i.d. Rayleigh distributed. $\gamma_{s r}, \gamma_{r d}$ and $\gamma_{s d}$ denote the instantaneous SNRs of S-R, R-D and S-D links. Due to Rayleigh fading, $\gamma_{s r}, \gamma_{r d}$ and $\gamma_{s d}$ are exponential random variables with expected values $\sigma_{s r}^{2}, \sigma_{r d}^{2}$ and $\sigma_{s d}^{2}$, respectively.

We assume that exact CSI is available at the receiver side for all S-D, S-R and R-D channels. The $\sigma_{s r}^{2}, \sigma_{r d}^{2}$ and $\sigma_{s d}^{2}$ can also change randomly reflecting distance dependent loss and shadowing but they are assumed to be known precisely at the relay and remain constant during the time of interest.

For simplicity, we assume a BPSK modulation for all the transmissions. However, the derivations can be extended to M-PSK modulation.

To facilitate the explanation, we assume a time-division protocol with two time slots. In the first slot the source transmits while the relay and the destination listens. In the second slot, there are two actions that can be taken by the relay node to minimize the expected end-to-end bit error rate with given observations: $a_{0}$, which represents remaining silent and $a_{1}$, which represents regenerating and retransmitting the signal. If the relay chooses to remain silent, the second time slot will be idle. In reality the idle slots can be skipped and the source can continue with the next data block until a change occurs in the channel states. If the relay chooses to retransmit, the destination combines the signals received in the first and the second time slots using maximal ratio combining (MRC).

\section{STATIC RELAYING}

In this protocol the decision of whether to use the relay or not is made based on the average channel gains, i.e. $\sigma_{s r}^{2}, \sigma_{r d}^{2}$ and $\sigma_{s d}^{2}$. This protocol represents the case where the relaying decisions are made at a higher layer for a time-scale much larger than the time scale of Rayleigh fading. In other words, the relay is either on or off as long as there are no major changes in the environment. Let $\mathrm{BER}^{s \rightarrow r}$ and $\mathrm{BER}^{s \rightarrow d}$ denote the bit error rates of S-R and S-D links:

$$
\mathrm{BER}^{s \rightarrow r}=\operatorname{BER}_{p 2 p}\left(\sigma_{s r}^{2}\right), \quad \operatorname{BER}^{s \rightarrow d}=\operatorname{BER}_{p 2 p}\left(\sigma_{s d}^{2}\right)
$$

where $\operatorname{BER}_{p 2 p}\left(\sigma^{2}\right)$ is the bit error rate of a point-to-point link using BPSK modulation under Rayleigh channel with an average $\mathrm{SNR}$ of $\sigma^{2}$. The $\operatorname{BER}_{p 2 p}\left(\sigma^{2}\right)$ is given by [7]:

$$
\operatorname{BER}_{p 2 p}\left(\sigma^{2}\right)=\frac{1}{2}\left(1-\sqrt{\frac{\sigma^{2}}{1+\sigma^{2}}}\right)
$$

The $\mathrm{BER}_{\text {coop }}$ denotes the probability of error at the destination after the maximal ratio combining (MRC) of the signals received from $S$ and $R$, given that the relay has correct detection. This is the well-known 2-branch diversity BER performance for BPSK in Rayleigh fading [7]:

$$
\begin{aligned}
& \operatorname{BER}_{\text {coop }}\left(\sigma_{r d}^{2}, \sigma_{s d}^{2}\right) \\
& =\left\{\begin{array}{l}
\frac{1}{4}\left(1-\sqrt{\frac{\sigma_{s d}^{2}}{1+\sigma_{s d}^{2}}}\right)^{2}\left(1+\frac{1}{2} \sqrt{\frac{\sigma_{s d}^{2}}{1+\sigma_{s d}^{2}}}\right), \sigma_{s d}^{2}=\sigma_{r d}^{2} \\
\frac{1}{2}\left[1-\frac{1}{\sigma_{r d}^{2}-\sigma_{s d}^{2}}\left(\sigma_{r d}^{2} \sqrt{\frac{\sigma_{r d}^{2}}{1+\sigma_{r d}^{2}}}-\sigma_{s d}^{2} \sqrt{\frac{\sigma_{s d}^{2}}{1+\sigma_{s d}^{2}}}\right)\right], \text { o.w. }
\end{array}\right.
\end{aligned}
$$

The $P_{\text {prop }}$ denotes the error propagation probability, which is the probability of bit error at the destination after MRC given that the relay has a bit error. We use the following approximate formula to calculate $P_{\text {prop }}$ for BPSK modulation:

$$
P_{p r o p}\left(\sigma_{r d}^{2}, \sigma_{s d}^{2}\right) \cong \frac{\sigma_{r d}^{2}}{\sigma_{r d}^{2}+\sigma_{s d}^{2}}
$$

The derivation of (4) is given in the Appendix. Throughout this paper, this approximation is used for all analytical developments.

If the relay does not transmit the e $2 \mathrm{e}$ bit error rate is equal to the bit error rate of direct $\mathrm{S}-\mathrm{D}$ transmission $\left(\mathrm{BER}^{s \rightarrow d}\right)$. If the relay always retransmits, we call this protocol fixed relaying. The e2e BER of fixed relaying is given by:

$$
\begin{aligned}
\operatorname{BER}_{e 2 e}^{f i x}\left(\sigma_{s r}^{2}, \sigma_{r d}^{2}, \sigma_{s d}^{2}\right) & =\left(1-\operatorname{BER}^{s \rightarrow r}\right) \operatorname{BER}_{c o o p}\left(\sigma_{r d}^{2}, \sigma_{s d}^{2}\right) \\
& +\operatorname{BER}^{s \rightarrow r} P_{p r o p}\left(\sigma_{r d}^{2}, \sigma_{s d}^{2}\right)
\end{aligned}
$$


Then, the optimal policy $\pi^{*}$ is to choose relaying if relaying results in a BER lower than the direct transmission.

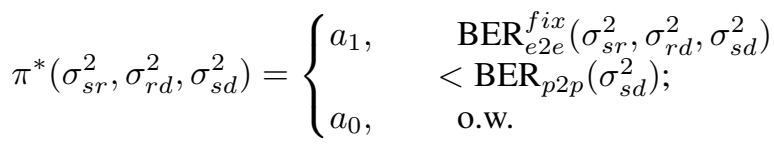

Hence, the minimum e2e error probability achieved by the optimal static relaying is:

$$
\begin{aligned}
& \operatorname{BER}^{e 2 e, s t}\left(\sigma_{s r}^{2}, \sigma_{r d}^{2}, \sigma_{s d}^{2}\right) \\
& =\min \left\{\operatorname{BER}_{e 2 e}^{f i x}\left(\sigma_{s r}^{2}, \sigma_{r d}^{2}, \sigma_{s d}^{2}\right), \operatorname{BER}_{p 2 p}\left(\sigma_{s d}^{2}\right)\right\}
\end{aligned}
$$

\section{DyNamic RELAYing}

In dynamic relaying the relay utilizes the instantaneous SNR of the S-R channel to make relaying decisions for each data block. Let $\pi\left(\gamma_{s r}, \sigma_{r d}^{2}, \sigma_{s d}^{2}\right)$ denote the relaying policy used by the relay. We denote the bit error probability achieved at given $\gamma_{s r}, \sigma_{r d}^{2}$ and $\sigma_{s d}^{2}$ values for a given action $a_{i}$ as $\operatorname{BER}^{e 2 e, d y n}\left(\gamma_{s r}, \sigma_{r d}^{2}, \sigma_{s d}^{2} ; a_{i}\right)$. The optimal policy that minimizes the average e2e bit error rate of dynamic relaying is given by

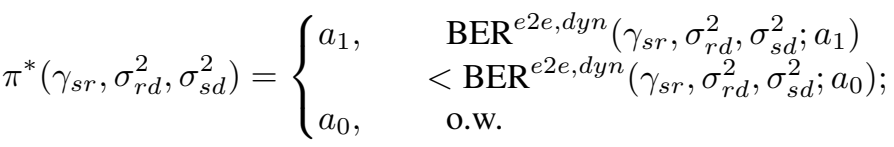

If the relay does not transmit, the e2e bit error probability for the block depends only on the direct channel:

$$
\operatorname{BER}^{e 2 e, d y n}\left(\gamma_{s r}, \sigma_{r d}^{2}, \sigma_{s d}^{2} ; a_{0}\right)=\operatorname{BER}^{s \rightarrow d}=\operatorname{BER}_{p 2 p}\left(\sigma_{s d}^{2}\right)
$$

If the relay transmits, the end-to-end BER is given by

$$
\begin{aligned}
& \operatorname{BER}^{e 2 e, d y n}\left(\gamma_{s r}, \sigma_{r d}^{2}, \sigma_{s d}^{2} ; a_{1}\right)= \\
& \mathbb{P}\left\{\text { no bit error at S-R link } \mid \gamma_{s r}\right\} \times \operatorname{BER}_{\text {coop }}\left(\sigma_{r d}^{2}, \sigma_{s d}^{2}\right) \\
& +\mathbb{P}\left\{\text { bit error at S-R link } \mid \gamma_{s r}\right\} P_{\text {prop }}\left(\sigma_{r d}^{2}, \sigma_{s d}^{2}\right)
\end{aligned}
$$

The probability of error of BPSK modulation for a given SNR is [7]:

$$
\mathbb{P}\left\{\text { bit error at S-R link } \mid \gamma_{s r}\right\}=Q\left(\sqrt{2 \gamma_{s r}}\right)
$$

Then by substituting (9) and (10) in the condition for $a_{1}$ in (8), we see that the relay retransmits if

$$
\begin{aligned}
\operatorname{BER}^{e 2 e, d y n}\left(\gamma_{s r}, \sigma_{r d}^{2}, \sigma_{s d}^{2} ; a_{1}\right) & <\mathrm{BER}^{e 2 e, d y n}\left(\gamma_{s r}, \sigma_{r d}^{2}, \sigma_{s d}^{2} ; a_{0}\right) \\
\rightarrow Q\left(\sqrt{2 \gamma_{s r}}\right) & <\delta\left(\sigma_{r d}^{2}, \sigma_{s d}^{2}\right)
\end{aligned}
$$

where $\delta$ is defined as

$$
\delta\left(\sigma_{r d}^{2}, \sigma_{s d}^{2}\right) \triangleq \frac{\operatorname{BER}_{p 2 p}\left(\sigma_{s d}^{2}\right)-\operatorname{BER}_{c o o p}\left(\sigma_{r d}^{2}, \sigma_{s d}^{2}\right)}{P_{p r o p}\left(\sigma_{r d}^{2}, \sigma_{s d}^{2}\right)-\operatorname{BER}_{c o o p}\left(\sigma_{r d}^{2}, \sigma_{s d}^{2}\right)}
$$

Then, we can simplify (8) into:

$$
\pi^{*}\left(\gamma_{s r}, \sigma_{r d}^{2}, \sigma_{s d}^{2}\right)= \begin{cases}a_{1}, & \gamma_{s r}>\gamma_{t}\left(\sigma_{r d}^{2}, \sigma_{s d}^{2}\right) ; \\ a_{0}, & \text { o.w. }\end{cases}
$$

where

$\gamma_{t}\left(\sigma_{r d}^{2}, \sigma_{s d}^{2}\right)= \begin{cases}0.5\left(Q^{-1}\left(\delta\left(\sigma_{r d}^{2}, \sigma_{s d}^{2}\right)\right)\right)^{2}, & \delta\left(\sigma_{r d}^{2}, \sigma_{s d}^{2}\right)<0.5 ; \\ 0, & \text { o.w. }\end{cases}$ and $Q^{-1}$ denotes the inverse $Q$ function. From (13) and (14), it clear that the optimal relaying strategy is a simple threshold rule on the instantaneous SNR of the S-R channel. The optimal threshold is function of two parameters only: average SNR of R-D and S-D channels. We note that for any $\sigma_{r d}^{2}, \sigma_{s d}^{2}>0$

$$
\operatorname{BER}_{\text {coop }}\left(\sigma_{r d}^{2}, \sigma_{s d}^{2}\right)<\operatorname{BER}_{p 2 p}\left(\sigma_{s d}^{2}\right)<P_{\text {prop }}\left(\sigma_{r d}^{2}, \sigma_{s d}^{2}\right) \text {. }
$$

Hence, $\delta\left(\sigma_{r d}^{2}, \sigma_{s d}^{2}\right)<1$ and $Q^{-1}\left(\delta\left(\sigma_{r d}^{2}, \sigma_{s d}^{2}\right)\right)$ is defined for any positive $\sigma_{s d}^{2}$ and $\sigma_{r d}^{2}$. Moreover, for positive $\sigma_{s d}^{2}$ and $\sigma_{r d}^{2}$ values (14) always gives non-negative threshold values.

1) The E2E Bit Error Rate (BER) Calculation: First we study a hypothetical system where the relay can detect all the bit errors and transmits each symbol only if it is detected correctly. We call this protocol as the genie-aided relaying protocol. The e2e BER of the genie-aided relaying protocol is equal to ${ }^{1}$

$$
\begin{aligned}
\operatorname{BER}_{e 2 e}^{g e n}\left(\sigma_{s r}^{2}, \sigma_{r d}^{2}, \sigma_{s d}^{2}\right) & =\left(1-\mathrm{BER}^{s \rightarrow r}\right) \mathrm{BER}_{c o o p} \\
& +\mathrm{BER}^{s \rightarrow r} \mathrm{BER}^{s \rightarrow d}
\end{aligned}
$$

where $\mathrm{BER}^{s \rightarrow r}$ and $\mathrm{BER}^{s \rightarrow d}$ are as given in (1). We note that the genie-aided relaying protocol constitutes a performance upper bound for the uncoded relaying protocols described in Section II.

Since the value of the threshold is independent of $\gamma_{s r}$, the average e2e bit error probability of dynamic relaying is given as follows:

$$
\begin{aligned}
& \operatorname{BER}_{e 2 e}^{d y n}\left(\sigma_{s r}^{2}, \sigma_{r d}^{2}, \sigma_{s d}^{2}\right)=\mathbb{P}\left\{\gamma_{s r} \leq \gamma_{t}\right\} \mathrm{BER}^{s \rightarrow d} \\
+ & \mathbb{P}\left\{\gamma_{s r}>\gamma_{t}\right\}\left(\mathrm{BER}_{t} P_{\text {prop }}+\left(1-\mathrm{BER}_{t}\right) \mathrm{BER}_{\text {coop }}\right) \\
= & \left(1-e^{-\gamma_{t} / \sigma_{s r}^{2}}\right) \mathrm{BER}^{s \rightarrow d} \\
+ & e^{-\gamma_{t} / \sigma_{s r}^{2}}\left(\mathrm{BER}_{t} P_{\text {prop }}+\left(1-\mathrm{BER}_{t}\right) \mathrm{BER}_{\text {coop }}\right)
\end{aligned}
$$

where $\mathrm{BER}_{t}$ is the bit error rate in S-R link given that $\gamma_{s r}>\gamma_{t}$. From the formulation of [3]:

$$
\begin{aligned}
\operatorname{BER}_{t}\left(\gamma_{t}, \sigma_{s r}^{2}\right) & =Q\left(\sqrt{2 \gamma_{t}}\right) \\
& -e^{\gamma_{t} / \sigma_{s r}^{2}} \sqrt{\frac{\sigma_{s r}^{2}}{1+\sigma_{s r}^{2}}} Q\left(\sqrt{2 \gamma_{t}\left(1+\frac{1}{\sigma_{s r}^{2}}\right)}\right)
\end{aligned}
$$

By substituting (1)-(4), (12), (14) and (17) in (16), we can analytically compute the average error performance of optimal dynamic relaying.

\section{NumericAl RESUlTS}

In Fig. 1, we plot the expression given in (14) for the optimal threshold and the threshold values obtained through numerical minimization of (16). We observe that the derived closed form expression fully agrees with the numerical results. It is also seen that the optimal threshold value is independent of the average SNR of the S-R link. Fig. 2 shows the e2e bit error rates obtained from (16) using (21). We note that although the optimal threshold is independent of $\sigma_{s r}^{2}$, the performance achieved by this threshold is a function of $\sigma_{s r}^{2}$.

${ }^{1}$ In the rest of the paper we drop the arguments of BER ${ }_{\text {coop }}$ and $P_{\text {prop }}$ to simplify the notation. 


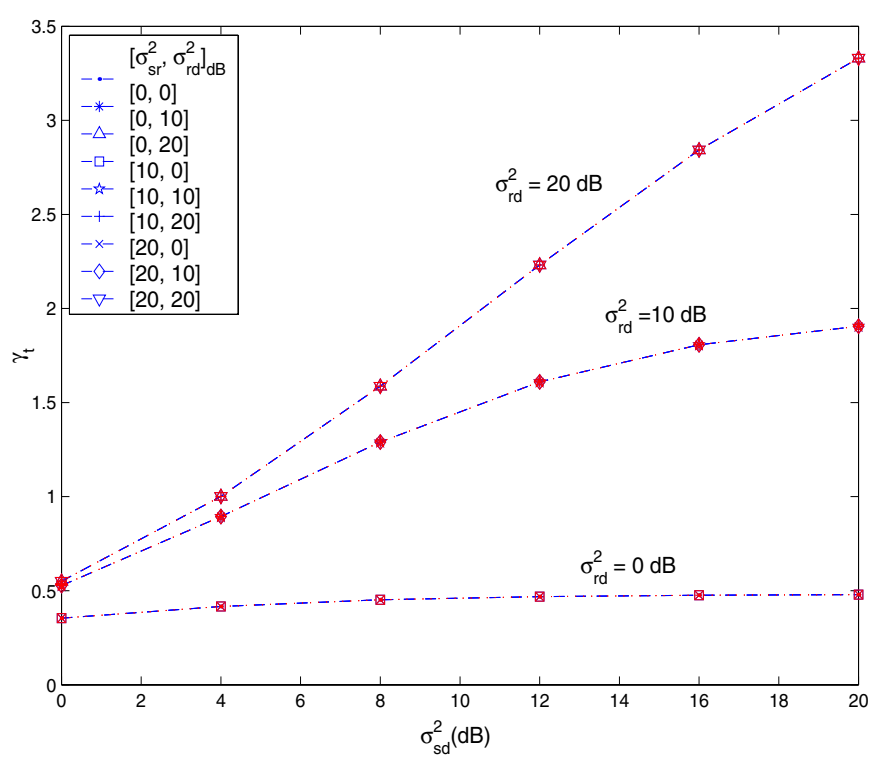

Fig. 1. Threshold values that minimize e2e BER given in (14) (dotted curves) and the thresholds obtained through numerical minimization of (16)(dashed curves) as a function of S-D SNR, for different S-R and R-D SNR values

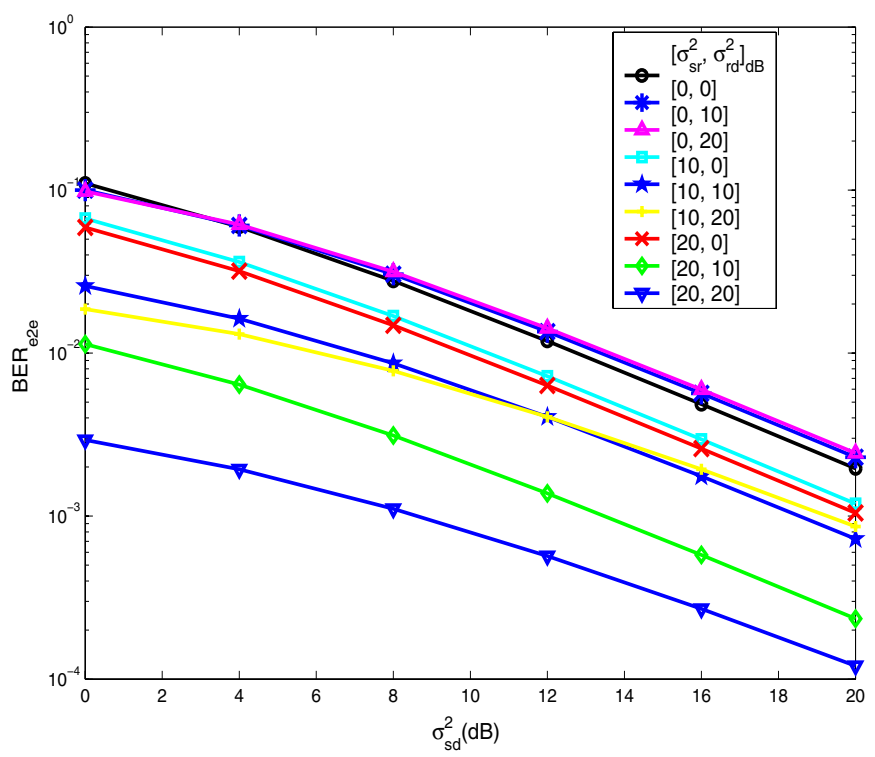

Fig. 2. The e2e BER of threshold relaying from 16) using the optimal threshold given in (14), as a function of S-D SNR, for different S-R and R-D SNR values

In order to understand the effect of relative channel qualities in the performance of static and dynamic relaying, we plot BER of these protocols as a function of the average SNR of S-R and R-D links. For this study, we keep the S-D link SNR constant at $10 \mathrm{~dB}$. Fig. 3 shows the performance of various protocols including fixed relaying and no relaying (i.e. only S-D transmission) as a function of $\sigma_{s r}^{2}$ for a weak and a strong R-D link. We observe that if R-D link is relatively poor $\left(\sigma_{r d}^{2}=0 \mathrm{~dB}\right)$, the performance is mainly constrained by the R-D link and all relaying protocols approach to the fixed relaying performance. However, when R-D link is strong $\left(\sigma_{r d}^{2}=20 \mathrm{~dB}\right)$ performance is determined by S-R link. In this
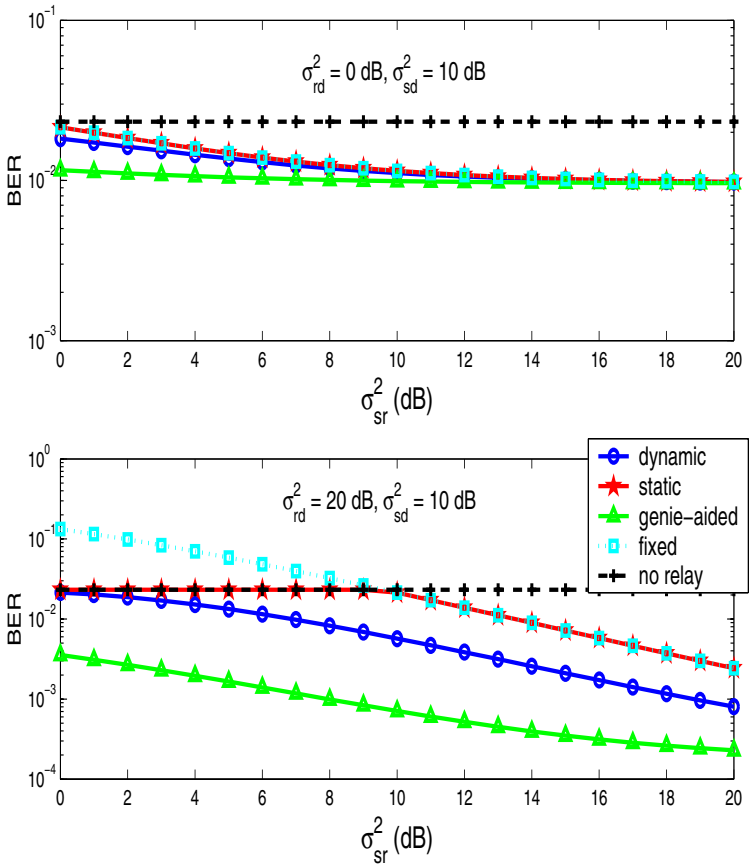

Fig. 3. The e2e BER comparison of all protocols for fixed $\sigma_{s d}^{2}=10 \mathrm{~dB}$ at two different $\sigma_{r d}^{2}$ values $(0$ and $20 \mathrm{~dB})$ as a function of $\sigma_{s r}^{2}$.
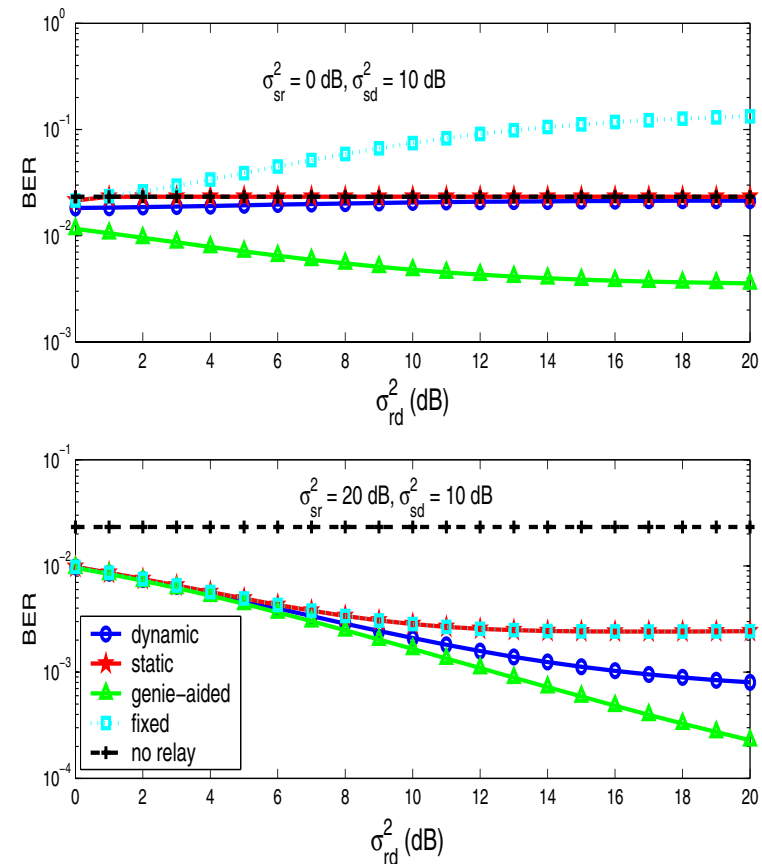

Fig. 4. The e2e BER comparison of all protocols for fixed $\sigma_{s d}^{2}=10 \mathrm{~dB}$ at two different $\sigma_{s r}^{2}$ values $\left(0\right.$ and $20 \mathrm{~dB}$ ) as a function of $\sigma_{r d}^{2}$.

case dynamic relaying is more efficient than static relaying since it can benefit from S-R link when it is reliable and prevent error propagation when it is not reliable. In Fig. 4, we study the effect of $\sigma_{r d}^{2}$ when the S-R channel is relatively weak $(0 \mathrm{~dB})$ and strong $(20 \mathrm{~dB})$. If S-R link is poor, both static and dynamic relaying protocols keep the relay silent most of the time. Hence, the performance is independent of 
R-D link and close to the performance of no relay case. An interesting observation from Fig. 4 is that the performance of fixed relaying deteriorates as R-D link improves due to the increase in $P_{\text {prop }}$. In the second case, where S-R link is strong, error events are rare at the relay. However, as R-D link gets stronger, the errors that occur at the relay are dominant at the destination. Dynamic relaying can further decrease error propagation by increasing the threshold as R-D link becomes stronger, which provides a performance advantage over static relaying.

\section{REMARKS AND CONCLUSIONS}

In this paper, we studied SNR-based selective digital relaying protocols where relaying decisions are made based on the state of the wireless links among the source, relay and the destination. We showed that if the relay is allowed to adapt according to each state of the source-relay channel, the optimal strategy to minimize the average bit error rate is a threshold rule. In contrast to the tendency in the literature to tie the threshold value to the average SNR of the source-relay channel, we showed that the optimal threshold is a function of the average SNR of source-destination and relay-destination links. In order to illustrate the benefits of dynamic relaying we analyzed another protocol, called static relaying, where the relaying decisions are based only on the average SNR values of all the links.

We evaluated the bit error performance of dynamic digital relaying and compared it to the static relaying protocol and a general performance upper bound for uncoded digital relaying protocols employing MRC at the destination. We showed that dynamic relaying using the optimal threshold value can take advantage of the source-relay channel adaptively providing significant performance advantage over static relaying.

\section{APPENDIX}

\section{ApProximation for $P_{\text {prop }}$}

Since we assume BPSK modulation, without loss of generality, we assume that the source sends +1 symbol, the relay sends -1 symbol and an error occurs if the destination decides that -1 is send. The received signals from the source and the relay are denoted by $y_{1}$ and $y_{2}$, respectively.

$$
\begin{aligned}
& y_{1}=\alpha_{s d} x+n_{1} \\
& y_{2}=-\alpha_{r d} x+n_{2}
\end{aligned}
$$

where $\alpha_{s d}$ and $\alpha_{r d}$ are the fading coefficients and $n_{1}$ and $n_{2}$ are i.i.d. Gaussian random variables with zero mean and $N_{0} / 2$ variance. After MRC, we can use the following decision variable

$$
\begin{aligned}
y_{n} & =\frac{\alpha_{s d}^{*}}{N_{0}} y_{1}+\frac{\alpha_{r d}^{*}}{N_{0}} y_{2} \\
& =\left(\frac{\left|\alpha_{s d}\right|^{2}}{N_{0}}-\frac{\left|\alpha_{r d}\right|^{2}}{N_{0}}\right) x+\frac{\alpha_{s d}^{*}}{N_{0}} n_{1}+\frac{\alpha_{r d}^{*}}{N_{0}} n_{2} \\
& =\left(\gamma_{s d}-\gamma_{r d}\right) x+\tilde{n}
\end{aligned}
$$

where we substituted $\gamma_{i j}=\left|\alpha_{i j}\right|^{2} / N_{0}$ and we defined $\tilde{n}=$ $\left(\alpha_{s d}^{*} / N_{0}\right) n_{1}+\left(\alpha_{r d}^{*} / N_{0}\right) n_{2}$. The effective noise $\tilde{n}$ is also a

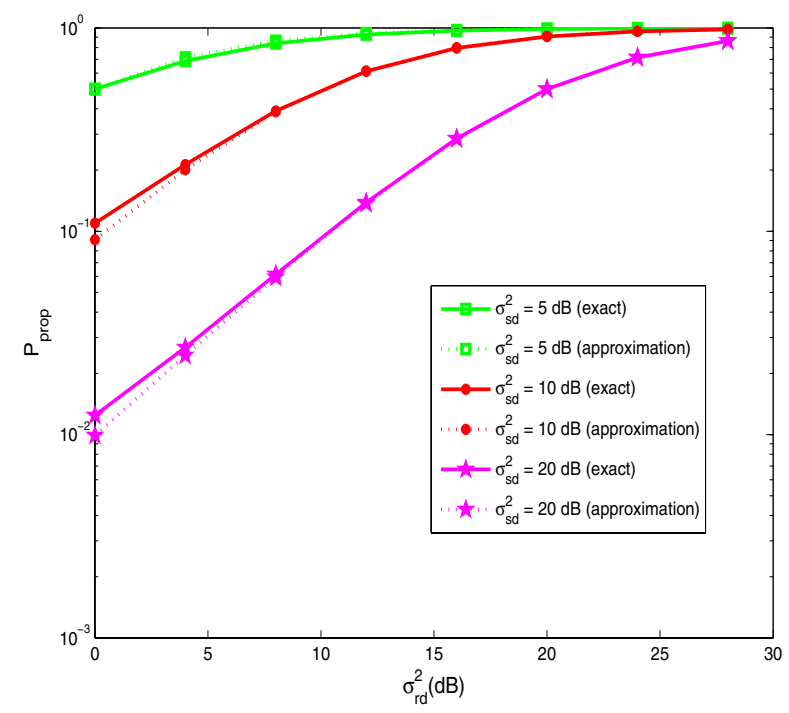

Fig. 5. Comparison of $P_{\text {prop }}$ values obtained from the approximation in (21) and from simulation as a function of $\sigma_{r d}^{2}$ for different $\sigma_{s d}^{2}$ values.

Gaussian random variable with zero mean and variance equal to $E\left[|\tilde{n}|^{2}\right]=\frac{1}{2}\left(\gamma_{s d}+\gamma_{r d}\right)$.

Since the destination assumes that both $\mathrm{S}$ and $\mathrm{R}$ send the same symbol, the optimal decision rule at the destination is:

$$
\hat{x}= \begin{cases}1, & y_{n} \geq 0 \\ -1, & y_{n}<0\end{cases}
$$

The probability of error propagation given the SNRs of R-D and S-D channels is

$$
\begin{aligned}
\mathbb{P}\left\{\text { error propagation } \mid \gamma_{r d}, \gamma_{s d}\right\} & =\mathbb{P}\left\{y_{n}<0 \mid \gamma_{r d}, \gamma_{s d}\right\} \\
& =\mathbb{P}\left\{\tilde{n}>\left(\gamma_{s d}-\gamma_{r d}\right) \mid \gamma_{r d}, \gamma_{s d}\right\} \\
& =Q\left(\frac{\gamma_{s d}-\gamma_{r d}}{\sqrt{\left(\gamma_{s d}+\gamma_{r d}\right) / 2}}\right)
\end{aligned}
$$

Then, $P_{\text {prop }}$ is given by

$$
\begin{aligned}
& P_{\text {prop }}=\iint \mathbb{P}\left\{\text { error propagation } \mid \gamma_{r d}, \gamma_{s d}\right\} p_{\gamma_{r d}}\left(\gamma_{r d}\right) \\
& \times p_{\gamma_{s d}}\left(\gamma_{s d}\right) d \gamma_{r d} d \gamma_{s d} \\
& =\int_{0}^{\infty} \int_{0}^{\infty} Q\left(\frac{\gamma_{s d}-\gamma_{r d}}{\sqrt{\left(\gamma_{s d}+\gamma_{r d}\right) / 2}}\right) \frac{1}{\sigma_{r d}^{2}} e^{-\gamma_{r d} / \sigma_{r d}^{2}} \\
& \times \frac{1}{\sigma_{s d}^{2}} e^{-\gamma_{s d} / \sigma_{s d}^{2}} d \gamma_{r d} d \gamma_{s d}
\end{aligned}
$$

Due to the complexity of the exact expression given in (19), we use a high SNR approximation for $P_{\text {prop }}$.

If we assume $\gamma_{s d}>>1$ and $\gamma_{r d}>1$ and $\gamma_{r d} / \gamma_{s d}=k$ $(0<k<\infty)$, then

$$
\begin{aligned}
Q\left(\frac{\gamma_{s d}-\gamma_{r d}}{\sqrt{\left(\gamma_{s d}+\gamma_{r d}\right) / 2}}\right) & =Q\left(\sqrt{\gamma_{s d}}\left(\frac{1-k}{\sqrt{(1+k) / 2}}\right)\right) \\
& \approx \begin{cases}1, & k \geq 1 ; \\
0, & k<1\end{cases}
\end{aligned}
$$


where, we use

$$
Q(x) \approx \begin{cases}1, & x>>1 \\ 0, & x<<1\end{cases}
$$

Substituting (20) in (19), we obtain

$$
\begin{aligned}
& P_{\text {prop }} \approx \mathbb{P}\{k>1\}=\mathbb{P}\left\{\gamma_{s d}<\gamma_{r d}\right\} \\
&= \int_{0}^{\infty} \int_{0}^{\gamma_{r d}} \frac{1}{\sigma_{s d}^{2}} \exp \left(-\gamma_{s d} / \sigma_{s d}^{2}\right) \\
& \quad \times \frac{1}{\sigma_{r d}^{2}} \exp \left(-\gamma_{r d} / \sigma_{r d}^{2}\right) d \gamma_{s d} d \gamma_{r d} \\
&=\frac{\sigma_{r d}^{2}}{\sigma_{s d}^{2}+\sigma_{r d}^{2}}
\end{aligned}
$$

In Fig. 5 we plot the probability of error propagation obtained both from simulations and from (21) in order to check the accuracy of our approximation at different $\sigma_{s d}^{2}$ and $\sigma_{r d}^{2}$ values. We observe that the analytical approximation is quite accurate for a wide range of $\sigma_{r d}^{2}$ and $\sigma_{s d}^{2}$ values.

\section{ACKNOWLEDGMENT}

The authors would like to thank Dr. Ian Marsland, Carleton University, for the useful discussions during the initial phase of this work.

\section{REFERENCES}

[1] N. Laneman, D. Tse, and G. Wornell, "Cooperative diversity in wireless networks: Efficient protocols and outage behavior," IEEE Trans. on Information Theory, vol. 50, pp. 3062-3080, Dec. 2004.

[2] A. Sendonaris, E. Erkip, and B. Aazhang, "User cooperation diversitypart I: System description," IEEE Trans. on Communications, vol. 51, pp. 1927-1938, November 2003 .

[3] P. Herhold, E. Zimmermann, and G. Fettweis, "A simple cooperative extension to wireless relaying," in Proceedings of International Zurich Seminar on Communications, 2004. Long version, available at http://wwwmns.ifn.et.tudresden.de/publications/2004/Herhold_P_IZS_04.pdf.

[4] A. Adinoyi and H. Yanikomeroglu, "Multi-antenna aspects of wireless fixed relays," in Proceedings of IEEE Wireless Communications and Networking Conference (WCNC 2006), 2006.

[5] W. S. W. P. Siriwongpairat, T. Himsoon and K. J. R. Liu, "Optimum threshold-selection relaying for decode-and-forward cooperation protocol," in Proceedings of IEEE Wireless Communications and Networking Conference (WCNC 2006), 2006.

[6] Z. Lin, E. Erkip, and A. Stefanov, "Cooperative regions and partner choice in coded cooperative systems," IEEE Transactions on Communications, vol. 54, July 2006.

[7] J. G. Proakis, Digital Communications. McGraw-Hill, 2000. 